\title{
Evidence-based use of cold for plantar fasciitis
}

\author{
Michael S. Laymon ${ }^{\mathrm{a}}$, Jerrold S. Petrofsky ${ }^{\mathrm{b}}$, Faris Alshammari ${ }^{\mathrm{b}}$, Stacy Fisher ${ }^{\mathrm{a}}$ \\ ${ }^{a}$ Department of Physical Therapy, Touro University Nevada, Henderson, NV, USA \\ ${ }^{b}$ Department of Physical Therapy, Loma Linda University, Loma Linda, CA, USA
}

Objective: The purpose of this study was to examine the effect of cold applied the night before or in the morning on pain and symptoms of plantar fasciitis.

Design: Experimental study.

Methods: Thirty subjects with plantar fasciitis were recruited for this study. Subjects with plantar fasciitis either had no intervention, cold applied (20 minutes) at night before bed, or 20 minutes in the morning upon wakening. Plantar fascia tenderness and pain were evaluated. There were ten subjects in each group. Measures included visual analog scale, plantar facial thickness via high resolution ultrasound, algometer measure, and range of motion of the ankle and foot. There were 3 groups of 10 subjects, control (no intervention), cold the night before bed, and cold in the morning before rising.

Results: The greatest relief of symptoms was cold used at bedtime the night before the measurements. Cold used in the morning was not as effective as cold used in the evening before bed. Cold use reduced the thickness of the plantar fascia and irritation. There was a $13 \%$ reduction in plantar fascia thickness with cold the night before $(p<0.05)$, a $44 \%$ reduction in pain and an $86 \%$ increase in the force that could be applied to the bottom of the foot without pain $(p<0.05)$.

Conclusions: Cold applied for 20 minutes prior bedtime is effective for reduced symptomology caused by plantar fascia inflammation.

Key Words: Cryotherapy, Foot, Pain, Plantar fasciitis

\section{Introduction}

Plantar fasciitis (PF) is a common disorder of the foot that affects millions of people [1,2]. It involves inflammation of the plantar fascia causing mild to severe pain when moving the foot or walking $[1,2]$. The plantar fascia is a thick fibrous connective tissue which originates at the medial tuberosity of the calcaneus and inserts into the proximal phalanges [3]. The central portion is the thickest, attaches at the posterior aspect of the medial tuberosity of the calcaneus posterior to the origin of the flexor digitorum brevis tendon, and is 1.5 to $2.0 \mathrm{~cm}$ in width, distally, at the level of the metatarso-phalangeal joints; the central portion of the plantar aponeurosis divides into five fascicles, one for each of the toes [4,5]. The lateral portion of the plantar aponeurosis arises from the lateral aspect of the medial tuberosity of the calcaneus and its distal medial and lateral bands attach to the plantar plate of the fourth toe and to the base of the fifth metatarsal respectively and is 1.0 to $1.5 \mathrm{~cm}$ in width. The medial portion of the plantar aponeurosis is thin and continuous medially with the dorsal fascia and laterally with the central portion of the plantar aponeurosis. Edema in the plantar fascia therefore effects movement in the foot by inhibiting normal tendon movement to flex and extend the foot $[4,5]$.

The etiology is quite variable [5-7] and includes ageing [6-9], abnormal foot positioning, increased body mass index more than 30, tight Achilles tendon, poor footwear, high intensity athletic activity and repetitive trauma and diabetes

Received: 9 November, 2013 Revised: 14 December, 2013 Accepted: 16 December, 2013

Corresponding author: Michael S. Laymon

Department of Physical Therapy, Touro University Nevada, 874 American Pacific Dr. Henderson, NV 89014, USA

Tel: 1-702-777-3051 Fax: 1-702-777-3051 E-mail: Michael.Laymon@tun.touro.edu

(c) This is an Open-Access article distributed under the terms of the Creative Commons Attribution Non-Commercial License (http://creativecommons.org/licens es/by-nc/3.0) which permits unrestricted non-commercial use, distribution, and reproduction in any medium, provided the original work is properly cited.

Copyright $@ 2013$ Korean Academy of Physical Therapy Rehabilitation Science 
[8-10]. Other inflammatory conditions can also contribute to this disorder. It is generally treated by stretching, heating with ultrasound, and even steroid injections [9]. But even with these modalities, the inflammation can return and severely limit normal gait.

However, one recurrent problem is that with normal conservative treatment for this disorder, modalities are mixed. What this means is that a physician or physical therapist will manipulate the foot by stretching and apply heat or cold and use non-steroidal anti-inflammatories or steroids and orthotics. Thus, if the inflammation gets better, there is no way of knowing why. It may be one modality, the combination of all interventions, or just normal healing.

In the present investigation we used cold as the only intervention and measured plantar fascia inflammation, pain level, tolerance to pressure on the plantar surface, and a standardized functional assessment instrument. Cold was applied for 20 minutes either at night at bedtime or first thing in the morning before weight bearing.

\section{Methods}

\section{Subjects}

Thirty subjects were recruited for this study. Eligible subjects were required to meet all the following inclusion criteria: (1) subjects of both genders are diagnosed with PF, (2) subject's age is between 18-65 years, (3) duration of symptoms is less than 5 years, and (4) plantar fascia thickness greater than $4 \mathrm{~mm}$ as assessed by imaging ultrasound. Exclusion criteria included: (1) previous fracture or surgery to the foot or (2) specific metabolic and connective tissue disorder associated with or contributing to the diagnosis of PF. All purposes and procedures were approved by the institutional review board (IRB) and each patient signed a statement of informed consent. The demographics of the subjects are listed in Table 1. There is a statistical difference

Table 1. General characteristics of subjects

$(\mathrm{N}=30)$

\begin{tabular}{lccc}
\hline \multicolumn{1}{c}{ Characteristic } & $\begin{array}{c}\text { Control } \\
(\mathrm{n}=10)\end{array}$ & $\begin{array}{c}\text { Cold night } \\
(\mathrm{n}=10)\end{array}$ & $\begin{array}{c}\text { Cold morning } \\
(\mathrm{n}=10)\end{array}$ \\
\hline Age $(\mathrm{yr})$ & $51.9(3.6)$ & $52.3(5.5)$ & $43.8(9.5)$ \\
Height $(\mathrm{cm})$ & $155.2(26.5)$ & $164.1(4.5)$ & $171.4(6.7)$ \\
Weight $(\mathrm{kg})$ & $85.4(15.8)$ & $75.8(19.1)$ & $81.3(19.0)$ \\
BMI & $29.4(4.2)$ & $27.9(5.8)$ & $27.5(5.2)$ \\
FAAM & $30.2(8.5)$ & $21.9(5.4)$ & $23.8(16.9)$ \\
\hline
\end{tabular}

Values are presented as mean (SD).

BMI: body mass index, FAAM: foot and ankle ability measure. in age between group 3 and groups 1 and 2. This study was approved by Solutions IRB.

\section{Visual analogue scale}

A visual analogue scale (VAS) was used to measure heel pain. It was a numerical scale $10 \mathrm{~cm}$ long where 0 indicated no pain, and 10 indicated the worst pain ever. The patient self-rated his/her foot pain based on his/her first steps in the morning, by placing a mark on the line representing his/her level of pain. This scale has been established as a reliable and valid instrument to measure acute and chronic pain.

\section{Pressure algometer}

A handheld pressure algometer was used to measure the heel tenderness threshold. Tenderness threshold was defined as the minimum pressure or force that produces pain. A pressure algometer is a force gauge fitted with a hard rubber tip and calibrated in $\mathrm{kg} / \mathrm{cm}^{2}$ (Wagner Inc., Greenwhich, CT, USA). To assess tenderness, the principal investigator first placed the patient in a supine position with legs fully extended and palpated and marked the tender point over the origin of the plantar fascia at the medial calcaneal tubercle. He then dorsiflexed the ankle and toes passively and applied the algometer over the mark placed on the medial calcaneal tubercle. The algometer contact head was aligned perpendicularly to the skin and the principal investigator gradually increased the algometer pressure until the patient reported pain. The algometer reading, which represented the pressure needed to elicit pain (in Newton per square centimeter), was recorded. Higher algometer scores indicated greater pressure tolerance and, hence, less tenderness. Lower algometer scores indicated less pressure tolerance and, thus, greater tenderness. The reliability and validity of pressure algometer as a measure of tenderness has been documented in the literature.

\section{Foot and ankle ability pain in activities of daily living}

To assess functional activities levels, the subjects were asked to record their ability to perform daily activities using the activities of daily living (ADL) subscale of the foot and ankle ability measure (FAAM). The ADL/FAAM identifies 21 daily activities, and participants rated their ability to complete each activity based on a scale ranging from no difficulty to inability to complete. Individual participant responses to the ADL/FAAM questions were converted to numerical scores using a 5-point scale, with scale ranging from 0 "no difficulty" to 4 "unable to do," certain daily activity. 
Thus a lower ADL/FAAM score indicates a higher functional activity level. FAAM is a self-reported instrument specific to those with lower leg musculoskeletal disorders. FAAM is a reliable, valid, and responsive self-reported instrument for assessing the activity and function level for patients with lower leg musculoskeletal disorders.

\section{Ultrasound}

Musculoskeletal ultrasound is a useful imaging tool in confirming a diagnosis of PF by measuring plantar fascia thickness before and after a given treatment regimen to gauge the treatment's efficacy. The standard "normal" or asymptomatic thickness value reported for the plantar fascia is 2.3 to $4.0 \mathrm{~mm}$, averaging $3.4 \mathrm{~mm}$. It is accepted that a thickness of greater than $4 \mathrm{~mm}$ would be consistent with PF. Each involved foot was evaluated sonographically with a L14-6 MHz linear array transducer (Mindray-M7 diagnostic ultrasound system) and acoustic coupling gel applied to the plantar surface of the foot. The plantar fascia is best examined with the patient in the prone position, with the affected foot hanging over the edge of the examination table with his or her ankle in neutral position. The ultrasound probe was applied vertically to the plantar aspect of the heel. The sagittal thickness of the proximal insertion of the plantar fascia was measured, at a standard reference point $5 \mathrm{~mm}$ from the proximal insertion at the anterior aspect of the inferior border of the calcaneus. A typical picture is shown in Figure 1.

\section{Applying cold to the foot}

Cold was applied by a ThermaCare (Pfizer Consumer Healthcare, NY, USA) cold wrap as described by the manu-

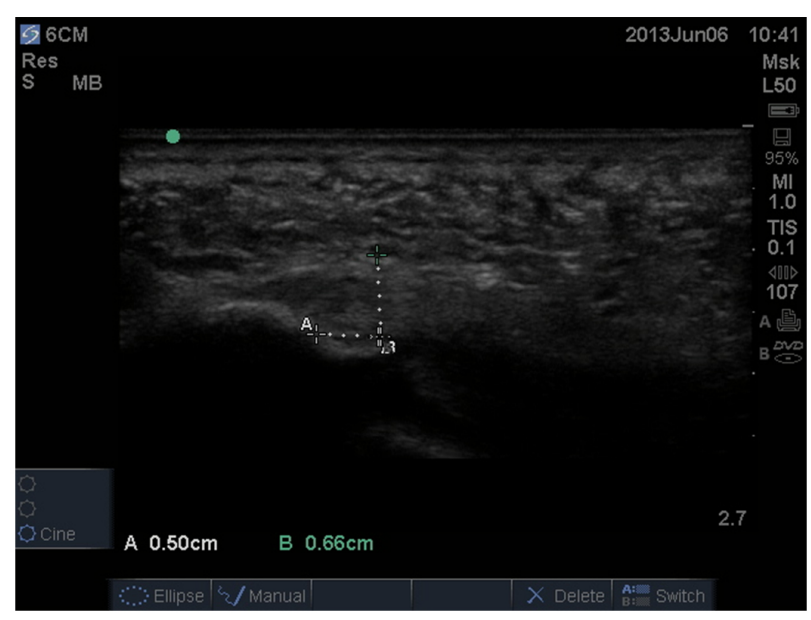

Figure 1. Typical subject pre modality. facturer for 20 minutes.

\section{Procedures}

The baseline evaluation included the measurement of: (1) heel pain with the VAS, (2) heel tenderness with the pressure algometer, (3) disability level, and (4) sagittal thickness of proximal plantar fascia.

The subject was assigned to one of 3 groups. One group was the control and only had measurements taken 2 days apart without intervention. The pre and post evaluation included: (1) heel pain; (2) heel tenderness; (3) disability level using ADL/FAAM, and (4) sagittal thickness of proximal plantar fascia with the ultrasound transducer. For the other 2 groups, the same measures were taken but an intervention was provided between the measures. In different groups, cold was used either the night before or at morning. All plantar fascia thickness measures were taken first thing in the morning for all groups.

\section{Data analysis}

Analysis of variance (ANOVA) test was used to determine if significant differences exist among the groups in terms of pain, tenderness, ADL, and fascia thickness. The level of significance was set at $p<0.05$.

\section{Results}

As seen here the measures were made $5 \mathrm{~cm}$ from the calcaneus and then the full thickness width was measured, which was here, $0.66 \mathrm{~cm}$. A typical subject before cold is shown in Figure 2. Figure 2 shows the pre data where the

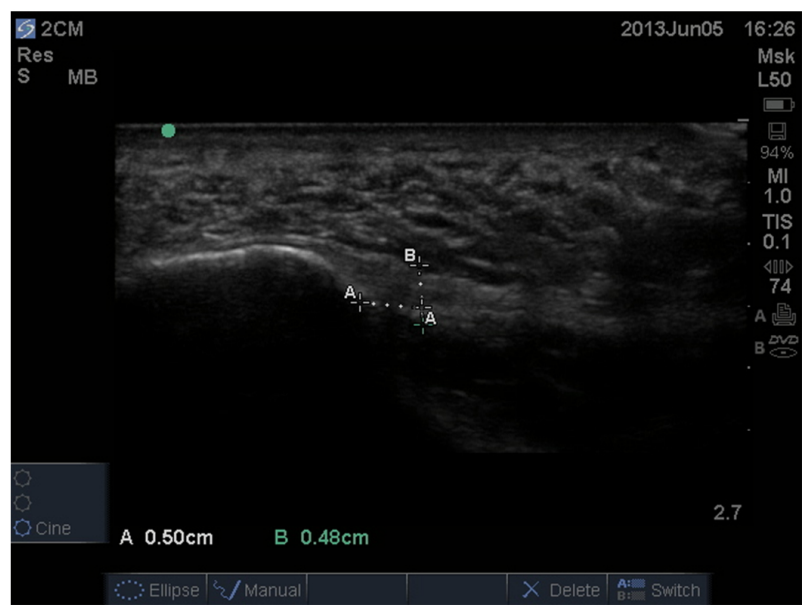

Figure 2. A subject before application of cold. 


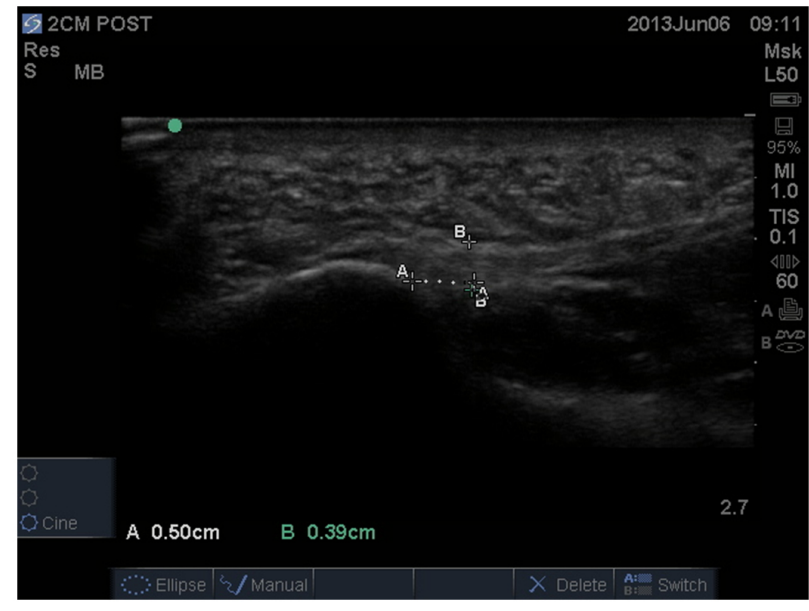

Figure 3. A subject after cold application.

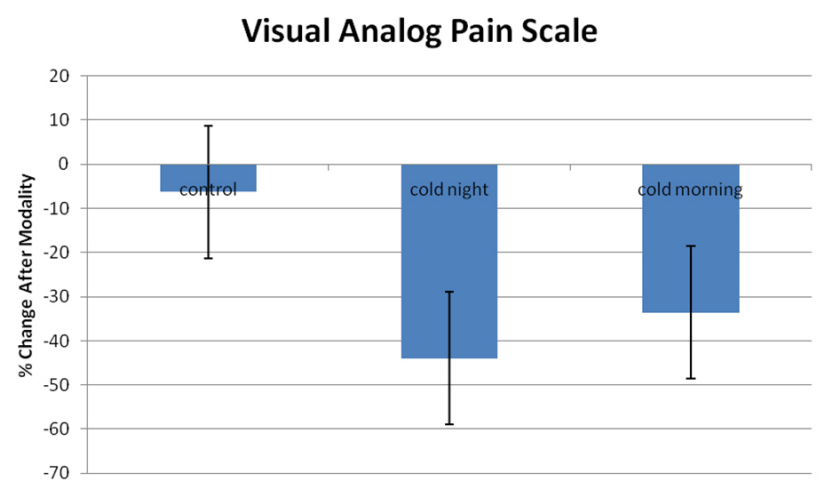

Figure 4. Percent change in the analog visual pain scale from the first to the second day after application of each modality. The data is the mean of 10 subjects $+/-$ the SD.

thickness of the plantar fascia was $0.48 \mathrm{~cm}$ whereas post cold, it was reduced to $0.39 \mathrm{~cm}$ (Figure 3).

As shown in Figure 4, cold applied at night before bed clearly produced the greatest reduction in pain the next morning. Cold at night and in the morning were significantly better than the control subject data $(p<0.01)$. The control data was not significantly different in the first and second measurements.

A similar change is seen in Figure 5. The greatest reduction in the thickness of the plantar fascia was with cold applied at bedtime. Cold applied the night before for $20 \mathrm{mi}-$ nutes had a carryover effect of a reduction in plantar fascia thickness of $13 \%$ in the morning. This was a statistically significant decrease at $p<0.01$. Both cold groups showed a reduction in the size of the plantar fascia but the night data was significantly more than the morning cold group $(p<0.05)$. Thus cold needs time to have an effect and simple acute ad-

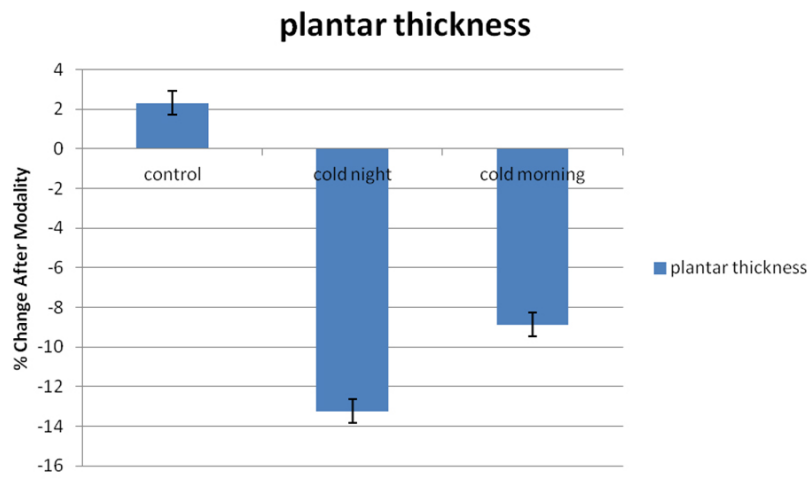

Figure 5. Change in plantar fascia thickness pre and post modality. The data is the mean of 10 subjects $+/-$ the SD.

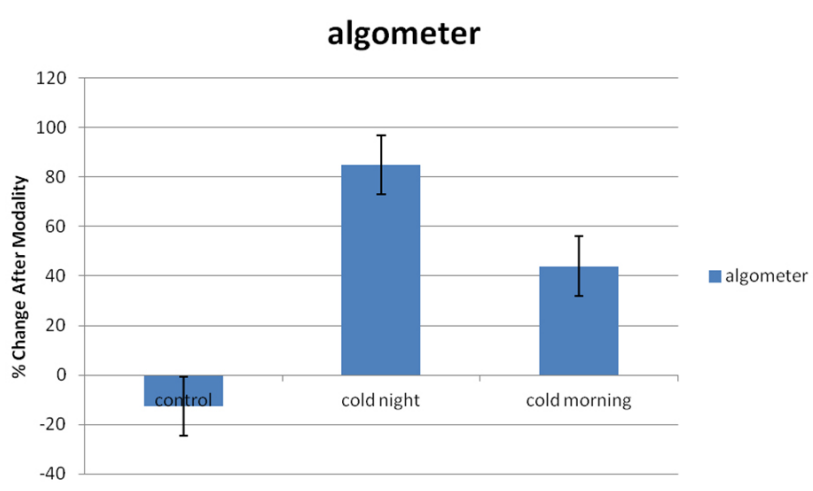

Figure 6. The change in algometer pressure for perception of pain before and after each modality in 10 subjects $+/-$ the SD.

ministration of cold was not as effective as cold the night before.

Figures 2 and 3 show the ultrasound recordings in a typical subject before and after cold. There was a clear reduction in plantar thickness. This reduced thickness also reduced tenderness in the area. The pressure applied to the plantar fascia with the algometer almost doubled when cold was used at night and was over $40 \%$ higher for cold used in the morning. For both cold groups the increase in pressure was significant $(p<0.01)$. There was no difference in the algometer data pre vs. post in the control group (Figure 6).

\section{Discussion}

$\mathrm{PF}$ is a common clinical disorder usually seen in people who walk or run incorrectly or are overweight $[11,12]$. "Classic" PF is intense, sharp heel pain with the first couple of steps in the morning primarily at the origin of the plantar fascia where it attaches to the anterior calcaneus [13]. This disorder costs between $\$ 192$ and \$376 million [14]. The 
plantar fascia can be easily overstressed. This can happen due to bone spurs (almost $50 \%$ of all cases) or other pathologies but the etiology is largely unknown [15]. The inflammation results in an increase in plantar facial thickness which can last for days or years or never fully heal [15]. Almost half of PF is due to heal spurs [16].

There have been many modalities used to treat PF. These include custom orthotic devices [15], rest, ice baths [13,14], heat, and stretching [15]. Night splinting and corticosteroid injections have also been used [17]. Heat is applied at night and in many cases has positive results but the results are unpredictable [18].

One method of examining PF is ultrasound [19]. It has been shown to be just as reliable as MRI in showing plantar swelling and thickness [19]. Power sonography has been shown to show vascularization, calcification and thickness of fascia and tendons [20]. Real time axial strain sonography has also been used to diagnose PF [21]. Studies clearly show thickening to over $6 \mathrm{~mm}$ in many patients with $\mathrm{PF}$ [22].

Heat has been tried in the past. Infrared heat, for example, was used for 6 months and only resulted in a reduction of $37 \%$ in the visual analog scale scores, a reduction similar to the one seen here but this one was after 1 treatment with ice [23]. Cold suffers from the same lack of evidence although web sites recommend cold or ice therapy. Furthermore, treatment is usually analyzed in terms of night splints, stretching, iontophoresis, low-energy extracorporeal shock waves, cold lasers and non-steroidal anti-inflammatories $[13,24]$. Very little attention has been paid to cold therapy applied in isolation to other interventions. Home modalities are largely ignored in the scientific literature.

Although there is a statistically significant difference in age between group 3 and groups 1 and 2, the actual age difference does not correlate to a difference in tissue metabolic or healing rates. The results for the use of cold for treatment of PF here are striking. This study investigated a single application of cold, further investigation is needed for multiple application.

Cold applied for 20 minutes prior bedtime is effective for reduced symptomology caused by plantar fascia inflammation.

\section{References}

1. Petrofsky JS, Alshammari F, Lee H, Yim JE, Bains G, Khowailed IA, et al. Electroencephalography to assess motor control during balance tasks in people with diabetes. Diabetes Technol Ther
2012;14:1068-76.

2. Yim J, Petrofsky J, Berk L, Daher N, Lohman E, Moss A, et al. Protective effect of anti-oxidants on endothelial function in young Korean-Asians compared to Caucasians. Med Sci Monit 2012;18:CR467-79.

3. Petrofsky JS. Resting blood flow in the skin: does it exist, and what is the influence of temperature, aging, and diabetes? J Diabetes Sci Technol 2012;6:674-85.

4. Bermudez LE, Inderlied CB, Kolonoski P, Chee CB, Aralar P, Petrofsky M, et al. Identification of (+)-erythro-mefloquine as an active enantiomer with greater efficacy than mefloquine against Mycobacterium avium infection in mice. Antimicrob Agents Chemother 2012;56:4202-6.

5. Petrofsky J, Berk L, Al-Nakhli H. The influence of autonomic dysfunction associated with aging and type 2 diabetes on daily life activities. Exp Diabetes Res 2012;2012:657103.

6. Petrofsky JS, Berk L, Alshammari F, Lee H, Hamdan A, Yim JE, et al. The interrelationship between air temperature and humidity as applied locally to the skin: the resultant response on skin temperature and blood flow with age differences. Med Sci Monit 2012;18:CR201-8.

7. Al-Nakhli HH, Petrofsky JS, Laymon MS, Berk LS. The use of thermal infra-red imaging to detect delayed onset muscle soreness. J Vis Exp 2012;(59).

8. Petrofsky J, Berk L, Alshammari F, Lee H, Hamdan A, Yim JE, et al. The effect of moist air on skin blood flow and temperature in subjects with and without diabetes. Diabetes Technol Ther 2012;14:105-16.

9. Al-Nakhli HH, Petrofsky JS, Laymon MS, Arai D, Holland K, Berk LS. The use of thermal infrared imaging to assess the efficacy of a therapeutic exercise program in individuals with diabetes. Diabetes Technol Ther 2012;14:159-67.

10. Petrofsky J. A method of measuring the interaction between skin temperature and humidity on skin vascular endothelial function in people with diabetes. J Med Eng Technol 2011;35:330-7.

11. Cutts S, Obi N, Pasapula C, Chan W. Plantar fasciitis. Ann R Coll Surg Engl 2012;94:539-42.

12. Scott RT, Hyer CF, Granata A. The correlation of Achilles tendinopathy and body mass index. Foot Ankle Spec 2013;6:283-5.

13. Young C. In the clinic. Plantar fasciitis. Ann Intern Med. 2012; 156:ITC1-1, ITC1-2, ITC1-3, ITC1-4, ITC1-5, ITC1-6, ITC1-7, ITC1-8, ITC1-9, ITC1-10, ITC1-11, ITC1-12, ITC1-13, ITC114, ITC1-15; quiz ITC1-16.

14. Tong KB, Furia J. Economic burden of plantar fasciitis treatment in the United States. Am J Orthop (Belle Mead NJ) 2010;39: 227-31.

15. Goff JD, Crawford R. Diagnosis and treatment of plantar fasciitis. Am Fam Physician 2011;84:676-82.

16. Barrett SL, Day SV, Pignetti TT, Egly BR. Endoscopic heel anatomy: analysis of 200 fresh frozen specimens. J Foot Ankle Surg 1995;34:51-6.

17. Akşahin E, Doğruyol D, Yüksel HY, Hapa O, Doğan O, Celebi L, et al. The comparison of the effect of corticosteroids and platelet-rich plasma (PRP) for the treatment of plantar fasciitis. Arch Orthop Trauma Surg 2012;132:781-5.

18. Gill LH, Kiebzak GM. Outcome of nonsurgical treatment for plantar fasciitis. Foot Ankle Int 1996;17:527-32.

19. Falsetti P, Frediani B, Acciai C, Baldi F, Filippou G, Prada EP, et 
al. Ultrasonographic study of Achilles tendon and plantar fascia in chondrocalcinosis. J Rheumatol 2004;31:2242-50.

20. Grassi W, Filippucci E. Is power Doppler sonography the new frontier in therapy monitoring? Clin Exp Rheumatol 2003;21: 424-8.

21. Sconfienza LM, Silvestri E, Orlandi D, Fabbro E, Ferrero G, Martini C, et al. Real-time sonoelastography of the plantar fascia: comparison between patients with plantar fasciitis and healthy control subjects. Radiology 2013;267:195-200.
22. Wearing SC, Smeathers JE, Sullivan PM, Yates B, Urry SR, Dubois P. Plantar fasciitis: are pain and fascial thickness associated with arch shape and loading? Phys Ther 2007;87:1002-8.

23. Reeboonlap N, Satitsmithpong N, Phisitkul P, Charakorn K. Outcome of plantar fasciitis treatment using monochrome infrared irradiation. J Med Assoc Thai 2012;95(Suppl 10):S147-50.

24. Crawford F, Thomson C. Interventions for treating plantar heel pain. Cochrane Database Syst Rev 2003;(3):CD000416. 